\title{
Strong Convergence of a New Iterative Method for Infinite Family of Generalized Equilibrium and Fixed-Point Problems of Nonexpansive Mappings in Hilbert Spaces
}

\author{
Shenghua Wang ${ }^{1,2}$ and Baohua Guo ${ }^{1,2}$ \\ ${ }^{1}$ National Engineering Laboratory for Biomass Power Generation Equipment, \\ North China Electric Power University, Baoding 071003, China \\ ${ }^{2}$ Department of Mathematics and Physics, North China Electric Power University, Baoding 071003, China
}

Correspondence should be addressed to Shenghua Wang, sheng-huawang@hotmail.com

Received 15 October 2010; Accepted 18 November 2010

Academic Editor: Qamrul Hasan Ansari

Copyright (C 2011 S. Wang and B. Guo. This is an open access article distributed under the Creative Commons Attribution License, which permits unrestricted use, distribution, and reproduction in any medium, provided the original work is properly cited.

\begin{abstract}
We introduce an iterative algorithm for finding a common element of the set of solutions of an infinite family of equilibrium problems and the set of fixed points of a finite family of nonexpansive mappings in a Hilbert space. We prove some strong convergence theorems for the proposed iterative scheme to a fixed point of the family of nonexpansive mappings, which is the unique solution of a variational inequality. As an application, we use the result of this paper to solve a multiobjective optimization problem. Our result extends and improves the ones of Colao et al. (2008) and some others.
\end{abstract}

\section{Introduction}

Let $H$ be a real Hilbert space and $T$ be a mapping of $H$ into itself. $T$ is said to be nonexpansive if

$$
\|T x-T y\| \leq\|x-y\|, \quad \forall x, y \in H .
$$

If there exists a point $u \in H$ such that $T u=u$, then the point $u$ is called a fixed point of $T$. The set of fixed points of $T$ is denoted by $F(T)$. It is well known that $F(T)$ is closed convex and also nonempty if $T$ has a bounded trajectory (see [1]). 
Let $f: H \rightarrow H$ be a mapping. If there exists a constant $0 \leq \kappa<1$ such that

$$
\|f x-f y\| \leq \kappa\|x-y\|, \quad \forall x, y \in H,
$$

then $f$ is called a contraction with the constant $\kappa$. Recall that an operator $A: H \rightarrow H$ is called to be strongly positive with coefficient $\bar{\gamma}>0$ if

$$
\langle A x, x\rangle \geq \bar{r}\|x\|^{2}, \quad \forall x \in H .
$$

Let $u \in H$ be a fixed point, $A$ be a strongly positive linear bounded operator on $H$ and $\{T\}_{n=1}^{N}$ be a finite family of nonexpansive mappings of $H$ into itself such that $F=$ $\bigcap_{n=1}^{N} F\left(T_{n}\right) \neq \emptyset$.

In 2003, Xu [2] introduced the following iterative scheme:

$$
x_{n+1}=\left(I-\epsilon_{n+1} A\right) T_{n+1} x_{n}+\epsilon_{n+1} u, \quad \forall n \geq 1,
$$

where $I$ is the identical mapping on $H$ and $T_{n}=T_{n \bmod N}$, and proved some strong convergence theorems for the iterative scheme to the solution of the quadratic minimization problem

$$
\min _{x \in F} \frac{1}{2}\langle A x, x\rangle-\langle x, u\rangle
$$

under suitable hypotheses on $\epsilon_{n}$ and the additional hypothesis:

$$
F=F\left(T_{1} T_{2} \cdots T_{N}\right)=F\left(T_{N} T_{1} \cdots T_{N-1}\right)=\cdots=F\left(T_{2} T_{3} \cdots T_{N} T_{1}\right) .
$$

Recently, Marino and Xu [3] introduced a new iterative scheme from an arbitrary point $x_{0} \in H$ by the viscosity approximation method as follows:

$$
x_{n+1}=\epsilon_{n} \gamma f\left(x_{n}\right)+\left(I-\epsilon_{n} A\right) T x_{n}, \quad \forall n \geq 1,
$$

and prove that the scheme strongly converges to the unique solution $x^{*}$ of the variational inequality:

$$
\left\langle(A-\gamma f) x^{*}, x-x^{*}\right\rangle \geq 0, \quad \forall x \in F(T)
$$

which is the optimality condition for the minimization problem:

$$
\min _{x \in F} \frac{1}{2}\langle A x, x\rangle-h(x),
$$

where $h$ is a potential function for $\gamma f$ (i.e., $h^{\prime}(x)=\gamma f(x)$ for all $x \in H$ ). 
Let $\left\{T_{n}\right\}_{n=1}^{N}$ be a finite family of nonexpansive mappings of $H$ into itself. In 2007, Yao [4] defined the mappings

$$
\begin{aligned}
U_{n, 1} & =\lambda_{n, 1} T_{1}+\left(1-\lambda_{n, 1}\right) I, \\
U_{n, 2} & =\lambda_{n, 2} T_{2} U_{n, 1}+\left(1-\lambda_{n, 2}\right) I, \\
& \vdots \\
U_{n, N-1}= & \lambda_{n, N-1} T_{N-1} U_{n, N-2}+\left(1-\lambda_{n, N-1}\right) I, \\
W_{n} & \equiv U_{n, N}=\lambda_{n, N} T_{N} U_{n, N-1}+\left(1-\lambda_{n, N}\right) I
\end{aligned}
$$

and, by extending (1.10), proposed the iterative scheme:

$$
x_{n+1}=\epsilon_{n} \gamma f\left(x_{n}\right)+\beta x_{n}+\left((1-\beta) I-\epsilon_{n} A\right) W_{n} x_{n}, \quad \forall n \geq 1 .
$$

Then he proved that the iterative scheme (1.10) strongly converges to the unique solution $x^{*}$ of the variational inequality:

$$
\left\langle(A-\gamma f) x^{*}, x-x^{*}\right\rangle \geq 0, \quad \forall x \in F,
$$

where $F=\bigcap_{n=1}^{N} F\left(T_{n}\right)$, which is the optimality condition for the minimization problem:

$$
\min _{x \in F} \frac{1}{2}\langle A x, x\rangle-h(x)
$$

where $h$ is a potential function for $\gamma f$ (However, Colao et al. pointed out in [5] that there is a gap in Yao's proof).

Let $C$ be a nonempty closed convex subset of $H$ and $G: C \times C \rightarrow \mathbb{R}$ be a bifunction. The equilibrium problem for the function $G$ is to determine the equilibrium points, that is, the set

$$
E P(G)=\{x \in C: G(x, y) \geq 0, \forall y \in C\}
$$

Let $A: C \rightarrow H$ be a nonlinear mapping. Let $E P(G, A)$ denote the set of all solutions to the following equilibrium problem:

$$
E P(\mathrm{cG}, A)=\{x \in C: G(x, y)+\langle A z, y-z\rangle \geq 0, \forall y \in C\}
$$

In the case of $A \equiv 0, E P(G, A)$ is deduced to $E P$. In the case of $G \equiv 0, E P(G, A)$ is also denoted by $\operatorname{VI}(C, A)$. 
In 2007, S. Takahashi and W. Takahashi [6] introduced a viscosity approximation method for finding a common element of $E P(G)$ and $F(T)$ from an arbitrary initial element $x_{1} \in H$

$$
\begin{gathered}
G\left(u_{n}, y\right)+\frac{1}{r_{n}}\left\langle y-u_{n}, u_{n}-x_{n}\right\rangle \geq 0, \quad \forall y \in C, \\
x_{n+1}=\epsilon_{n} f\left(x_{n}\right)+\left(1-\epsilon_{n}\right) T u_{n}, \quad \forall n \geq 1
\end{gathered}
$$

and proved that, under certain appropriate conditions over $\epsilon_{n}$ and $r_{n}$, the sequences $\left\{x_{n}\right\}$ and $\left\{u_{n}\right\}$ both converge strongly to $z=P_{F(T) \cap E P(G)} f(z)$.

By combing the schemes (1.7) and (1.16), Plubtieg and Punpaeng [7] proposed the following algorithm:

$$
\begin{gathered}
G\left(u_{n}, y\right)+\frac{1}{r_{n}}\left\langle y-u_{n}, u_{n}-x_{n}\right\rangle \geq 0, \quad \forall y \in C, \\
x_{n+1}=\epsilon_{n} \gamma f\left(x_{n}\right)+\left(I-\epsilon_{n} A\right) T u_{n}, \quad \forall n \geq 1,
\end{gathered}
$$

and proved that the iterative schemes $\left\{x_{n}\right\}$ and $\left\{u_{n}\right\}$ converge strongly to the unique solution $z$ of the variational inequality:

$$
\langle(A-\gamma f) z, x-z\rangle \geq 0, \quad \forall x \in F(T) \cap E P(G),
$$

which is the optimality condition for the minimization problem:

$$
\min _{x \in F(T) \cap E P(G)} \frac{1}{2}\langle A x, x\rangle-h(x)
$$

where $h$ is a potential function for $\gamma f$.

Very recently, for finding a common element of the set of a finite family of nonexpansive mappings and the set of solutions of an equilibrium problem, by combining the schemes (1.11) and (1.17), Colao et al. [5] proposed the following explicit scheme:

$$
\begin{gathered}
G\left(u_{n}, y\right)+\frac{1}{r_{n}}\left\langle y-u_{n}, u_{n}-x_{n}\right\rangle \geq 0, \quad \forall y \in C, \\
x_{n+1}=\epsilon_{n} \gamma f\left(x_{n}\right)+\beta x_{n}+\left((1-\beta) I-\epsilon_{n} A\right) W_{n} u_{n}, \quad \forall n \geq 1,
\end{gathered}
$$

and proved under some certain hypotheses that both sequences $\left\{x_{n}\right\}$ and $\left\{u_{n}\right\}$ converge strongly to a point $x^{*} \in F$ which is an equilibrium point for $G$ and is the unique solution of the variational inequality:

$$
\left\langle(A-\gamma f) x^{*}, x-x^{*}\right\rangle \geq 0, \quad \forall x \in F \cap E P(G),
$$

where $F=\bigcap_{n=1}^{N} F\left(T_{n}\right)$. 
The equilibrium problems have been considered by many authors; see, for example, $[6,8-19]$ and the reference therein. But, in these references, the authors only considered at most finite family of equilibrium problems and few of authors investigate the infinite family of equilibrium problems in a Hilbert space or Banach space. In this paper, we consider a new iterative scheme for obtaining a common element in the solution set of an infinite family of generalized equilibrium problems and in the common fixed-point set of a finite family of nonexpansive mappings in a Hilbert space. Let $\left\{T_{n}\right\}_{n=1}^{N}(N \geq 1)$ be a finite family of nonexpansive mappings of $H$ into itself, be $\left\{G_{n}\right\}: C \times C \rightarrow \mathbb{R}$ be an infinite family of bifunctions, and be $\left\{A_{n}\right\}: C \rightarrow H$ be an infinite family of $k_{n}$-inverse-strongly monotone mappings. Let $\left\{r_{n}\right\}$ be a sequence such that $r_{n} \subset\left(r, 2 k_{n}\right)$ with $r>0$ for each $n \geq 1$. Define the mapping $T_{r_{\mathrm{i}}}: H \rightarrow C$ by

$$
T_{r_{i}}(x)=\left\{z \in C: G_{i}(z, y)+\frac{1}{r_{i}}\langle y-z, z-x\rangle \geq 0, \forall y \in C\right\}, \quad x \in H, i \geq 1 .
$$

Assume that $\Omega=\bigcap_{i=1}^{N} F\left(T_{i}\right) \bigcap_{i=1}^{\infty} E P\left(G_{i}, A_{i}\right) \neq \emptyset$. For an arbitrary initial point $x_{1} \in H$, we define the iterative scheme $\left\{x_{n}\right\}$ by

$$
\begin{gathered}
z_{n}=\alpha_{n} x_{n}+\sum_{i=1}^{n}\left(\alpha_{i-1}-\alpha_{i}\right) T_{r_{i}}\left(I-r_{i} A_{i}\right) x_{n}, \\
x_{n+1}=\epsilon_{n} \gamma f\left(x_{n}\right)+\delta_{n} B x_{n}+\left(I-\delta_{n} B-\epsilon_{n} A\right) W_{n} z_{n}, \quad \forall n \geq 1,
\end{gathered}
$$

where $\alpha_{0}=1,\left\{\alpha_{n}\right\},\left\{\epsilon_{n}\right\}$ and $\left\{\delta_{n}\right\}$ are three sequences in $(0,1), A$ and $B$ are both strongly positive linear bounded operators on $H, W_{n}$ is defined by (1.10), and prove that, under some certain appropriate hypotheses on the control sequences, the sequence $\left\{x_{n}\right\}$ strongly converges to a point $x^{*} \in \Omega$, which is the unique solution of the variational inequality:

$$
\left\langle(A-\gamma f) x^{*}, x-x^{*}\right\rangle \geq 0, \quad \forall x \in \Omega .
$$

If $A_{i} \equiv A_{0}, G_{i} \equiv G$ and $r_{i} \equiv r$, then (1.23) is reduced to the iterative scheme:

$$
\begin{gathered}
z_{n}=\alpha_{n} x_{n}+\left(1-\alpha_{n}\right) T_{r}\left(I-r A_{0}\right) x_{n} \\
x_{n+1}=\epsilon_{n} \gamma f\left(x_{n}\right)+\delta_{n} B x_{n}+\left(I-\delta_{n} B-\epsilon_{n} A\right) W_{n} z_{n}, \quad \forall n \geq 1 .
\end{gathered}
$$

The proof method of the main result of this paper is different with ones of others in the literatures and our result extends and improves the ones of Colao et al. [5] and some others. 


\section{Preliminaries}

Let $C$ be a closed convex subset of a Hilbert space $H$. For any point $x \in H$, there exists a unique nearest point in $C$, denoted by $P_{C} x$, such that

$$
\left\|x-P_{C} x\right\| \leq\|x-y\|, \quad \forall y \in C
$$

Then $P_{C}$ is called the metric projection of $H$ onto $C$. It is well known that $P_{C}$ is a nonexpansive mapping of $H$ onto $C$ and satisfies the following:

$$
\left\langle x-y, P_{C} x-P_{C} y\right\rangle \geq\left\|P_{C} x-P_{C} y\right\|^{2}, \quad \forall x, y \in H .
$$

Let $A$ be a mapping from $C$ into $H$, then $A$ is called monotone if

$$
\langle x-y, A x-A y\rangle \geq 0
$$

for all $x, y \in C$. However, $A$ is called an $\alpha$-inverse-strongly monotone mapping if there exists a positive real number $\alpha$ such that

$$
\langle x-y, A x-A y\rangle \geq \alpha\|A x-A y\|^{2}
$$

for all $x, y \in C$. Let $I$ denote the identity mapping of $H$, then for all $x, y \in C$ and $\lambda>0$, one has [20]

$$
\|(I-\lambda A) x-(I-\lambda A) y\|^{2} \leq\|x-y\|^{2}+\lambda(\lambda-2 \alpha)\|A x-A y\|^{2} .
$$

Hence, if $\lambda \in(0,2 \alpha]$, then $I-\lambda A$ is a nonexpansive mapping of $C$ into $H$.

If there exists $u \in C$ such that

$$
\langle v-u, A u\rangle \geq 0
$$

for all $v \in C$, then $u$ is called the solution of this variational inequality. The set of all solutions of the variational inequality is denoted by $\mathrm{VI}(C, A)$.

In this paper, we need the following lemmas. 
Fixed Point Theory and Applications

Lemma 2.1 (see [21]). Given $x \in H$ and $y \in C$. Then $P_{C} x=y$ if and only if there holds the inequality

$$
\langle x-y, y-z\rangle \geq 0, \quad \forall z \in C
$$

Lemma 2.2 (see [22]). Let $\left\{s_{n}\right\}$ be a sequence of nonnegative real numbers satisfying

$$
s_{n+1} \leq\left(1-\eta_{n}\right) s_{n}+\eta_{n} \tau_{n}+\xi_{n}, \quad \forall n \geq 0,
$$

where $\left\{\eta_{n}\right\},\left\{\tau_{n}\right\}$, and $\left\{\xi_{n}\right\}$ satisfy the conditions:

(1) $\left\{\eta_{n}\right\} \subset[0,1], \sum_{n=1}^{\infty} \eta_{n}=\infty$ or, equivalently, $\prod_{n=0}^{\infty}\left(1-\eta_{n}\right)=0$;

(2) $\limsup _{n \rightarrow \infty} \tau_{n} \leq 0$;

(3) $\xi_{n} \geq 0(n \geq 0), \sum_{n=0}^{\infty} \xi_{n}<\infty$.

Then $\lim _{n \rightarrow \infty} s_{n}=0$.

Let $H$ be a Hilbert space. For all $x, y \in H$, the following equality holds:

$$
\|x+y\|^{2}=\|x\|^{2}+2\langle y, x+y\rangle-\|y\|^{2}
$$

Therefore, the following lemma naturally holds.

Lemma 2.3. Let $H$ be a real Hilbert space. The following identity holds:

$$
\|x+y\|^{2} \leq\|x\|^{2}+2\langle y, x+y\rangle, \quad \forall x, y \in H
$$

Lemma 2.4 (see [3]). Assume that $A$ is a strongly positive linear bounded operator on a Hilbert space $H$ with coefficient $\bar{\gamma}>0$ and $0<\rho \leq\|A\|^{-1}$. Then $\|I-\rho A\| \leq 1-\rho \bar{\gamma}$.

Lemma 2.5 (see [2]). Assume that $\left\{a_{n}\right\}$ is a sequence of nonnegative numbers such that

$$
a_{n+1} \leq\left(1-\gamma_{n}\right) a_{n}+\delta_{n}, \quad \forall n \geq 0,
$$

where $\left\{\gamma_{n}\right\}$ is a sequence in $(0,1)$ and $\delta_{n}$ is a sequence in $\mathbb{R}$ such that

$$
\begin{aligned}
& \text { (1) } \sum_{n=1}^{\infty} \gamma_{n}=\infty ; \\
& \text { (2) } \lim \sup _{n \rightarrow \infty}\left(\delta_{n} / \gamma_{n}\right) \leq 0 \text { or } \sum_{n=1}^{\infty}\left|\delta_{n}\right|<\infty
\end{aligned}
$$

Then $\lim _{n \rightarrow \infty} a_{n}=0$. 
Lemma 2.6 (see [23]). Let $C$ be a nonempty closed convex subset of a Hilbert space $H$ and let $G$ : $C \times C \rightarrow \mathbb{R}$ be a bifunction which satisfies the following:

(A1) $G(x, x)=0$ for all $x \in C$;

(A2) $G$ is monotone, that is, $G(x, y)+G(y, x) \leq 0$ for all $x, y \in C$;

(A3) For each $x, y, z \in C$,

$$
\lim _{t \downarrow 0} G(t z+(1-t) x, y) \leq G(x, y)
$$

(A4) For each $x \in C, y \mapsto G(x, y)$ is convex and lower semicontinuous.

For $x \in H$ and $r>0$, define a mapping $T_{r}: H \rightarrow C$ by

$$
T_{r}(x)=\left\{z \in C: G(z, y)+\frac{1}{r}\langle y-z, z-x\rangle \geq 0, \forall y \in C\right\} .
$$

Then $T_{r}$ is well defined and the following hold:

(1) $T_{r}$ is single-valued;

(2) $T_{r}$ is firmly nonexpansive, that is, for any $x, y \in H$,

$$
\left\|T_{r} x-T_{r} y\right\|^{2} \leq\left\langle T_{r} x-T_{r} y, x-y\right\rangle
$$

(3) $F\left(T_{r}\right)=\operatorname{EP}(G)$;

(4) $\operatorname{EP}(G)$ is closed and convex.

It is easy to see that if there exists some point $v \in C$ such that $v=T_{r}(I-r A) v$, where $A: C \rightarrow H$ is an $\alpha$-inverse strongly monotone mapping, then $v \in E P(G, A)$. In fact, since $v=T_{r}(I-r A) v$, one has

$$
G(v, y)+\frac{1}{r}\langle y-v, v-(I-r A) v\rangle \geq 0, \quad \forall y \in C
$$

that is,

$$
G(v, y)+\langle y-v, A v\rangle \geq 0, \quad \forall y \in C
$$

Hence, $v \in E P(G, A)$.

Let $C$ be a nonempty convex subset of a Banach space. Let $\left\{T_{i}\right\}_{i=1}^{N}$ be a finite family of nonexpansive mappings of $C$ into itself and $\lambda_{1}, \lambda_{2}, \ldots, \lambda_{N}$ be real numbers such that $0 \leq \lambda_{i} \leq 1$ 
for each $i=1,2, \ldots, N$. Define a mapping $W$ of $C$ into itself as follows:

$$
\begin{aligned}
U_{1} & =\lambda_{1} T_{1}+\left(1-\lambda_{1}\right) I, \\
U_{2}= & \lambda_{2} T_{2} U_{1}+\left(1-\lambda_{2}\right) I, \\
& \vdots \\
U_{N-1} & =\lambda_{N-1} T_{N-1} U_{N-2}+\left(1-\lambda_{N-1}\right) I, \\
W & =U_{N}=\lambda_{N} T_{N} U_{N-1}+\left(1-\lambda_{N}\right) I .
\end{aligned}
$$

Such a mapping $W$ is called the $W$-mapping generated by $T_{1}, T_{2}, \ldots, T_{N}$ and $\lambda_{1}, \lambda_{2}, \ldots, \lambda_{N}$ (see $[5,24,25])$.

Lemma 2.7 (see [26]). Let $C$ be a nonempty closed convex subset of a Banach space. Let $T_{1}, T_{2}, \ldots, T_{N}$ be nonexpansive mappings of $C$ into itself such that $\bigcap_{i=1}^{N} F\left(T_{i}\right) \neq \emptyset$ and let $\lambda_{1}, \lambda_{2}, \ldots, \lambda_{N}$ be real numbers such that $0<\lambda_{i}<1$ for each $i=1,2, \ldots, N-1$ and $0<\lambda_{N} \leq 1$. Let $W$ be the $W$-mapping of $C$ generated by $T_{1}, T_{2}, \ldots, T_{N}$ and $\lambda_{1}, \lambda_{2}, \ldots, \lambda_{N}$. Then $F(W)=\bigcap_{i=1}^{N} F\left(T_{i}\right)$.

Lemma 2.8 (see [5]). Let $C$ be a nonempty convex subset of a Banach space. Let $\left\{T_{i}\right\}_{i=1}^{N}$ be a finite family of nonexpansive mappings of $C$ into itself and let $\left\{\lambda_{n, i}\right\}_{i=1}^{N}$ be sequences in $[0,1]$ such that $\lambda_{n, i} \rightarrow \lambda_{i}$ for each $i=1,2, \ldots, N$. Moreover, for each $n \in \mathbb{N}$, let $W$ and $W_{n}$ be the $W$ mappings generated by $T_{1}, T_{2}, \ldots, T_{N}$ and $\lambda_{1}, \lambda_{2}, \ldots, \lambda_{N}$ and $T_{1}, T_{2}, \ldots, T_{N}$ and $\lambda_{n, 1}, \lambda_{n, 2}, \ldots, \lambda_{n, N}$, respectively. Then, for all $x \in C$, it follows that

$$
\lim _{n \rightarrow \infty}\left\|W_{n} x-W x\right\|=0
$$

\section{Main Results}

Now, we give our main results in this paper.

Theorem 3.1. Let $H$ be a Hilbert space and $C$ be a nonempty closed convex subset of $H$. Let $f: H \rightarrow$ $H$ be a contraction with coefficient $0<\kappa<1, A, B: H \rightarrow H$ be strongly positive linear bounded self-adjoint operators with coefficients $\bar{\gamma}>0$ and $\bar{\beta}>2\|B\|+\|B\|^{2}$, respectively, $\left\{T_{n}\right\}_{n=1}^{N}: H \rightarrow$ $H(N \geq 1)$ be a finite family of nonexpansive mappings, $\left\{G_{n}\right\}: C \times C \rightarrow \mathbb{R}$ be an infinite family of bifunctions satisfying $(A 1)-(A 4)$, and $\left\{A_{n}\right\}: C \rightarrow H$ be an infinite family of inverse-strongly monotone mappings with constants $\left\{k_{n}\right\}$ such that $\Omega=\left(\bigcap_{i=1}^{N} F\left(T_{i}\right)\right) \cap\left(\bigcap_{i=1}^{\infty} E P\left(G_{i}, A_{i}\right)\right) \neq \emptyset$. Let $\left\{\epsilon_{n}\right\}$ and $\left\{\delta_{n}\right\}$ be two sequences in $(0,1),\left\{\lambda_{n, i}\right\}_{i=1}^{N}$ be asequence in $[a, b]$ with $0<a \leq b<1,\left\{r_{n}\right\}$ be a sequence in $\left(r, 2 k_{n}\right)$ with $r>0$ and $\left\{\alpha_{n}\right\}$ be a strictly decreasing sequence $[0,1)$. Set $\alpha_{0}=1$. Take a fixed number $\gamma>0$ with $0<\bar{\gamma}-\gamma \kappa<1$. Assume that

(E1) $\lim _{n \rightarrow \infty} \epsilon_{n}=0$ and $\sum_{n=1}^{\infty} \epsilon_{n}=\infty$;

(E2) $\lim _{n \rightarrow \infty}\left|\lambda_{n+1, i}-\lambda_{n, i}\right|=0$ for each $i=1,2, \ldots, N$;

(E3) $0 \leq \delta_{n}+\epsilon_{n} \leq 1$ for all $n \geq 1$;

(E4) $\left\{\delta_{n}\right\} \subset\left[0, \min \left\{c, 1 / \beta,\left(2\|B\|+\|B\|^{2}-\bar{\beta}+\sqrt{\left(\bar{\beta}-\|B\|^{2}-2\|B\|\right)^{2}+8 \bar{\beta}\|B\|}\right) / 4 \bar{\beta}\|B\|\right\}\right)$ with $c<1$

(E5) $\sum_{n=1}^{\infty}\left|\epsilon_{n+1}-\epsilon_{n}\right|<\infty, \sum_{n=1}^{\infty}\left|\alpha_{n+1}-\alpha_{n}\right|<\infty, \quad \sum_{n=1}^{\infty}\left|\delta_{n+1}-\delta_{n}\right|<\infty$. 
Then the sequence $\left\{x_{n}\right\}$ defined by (1.23) converges strongly to $x^{*} \in \Omega$, which is the unique solution of the variational inequality: (1.24), that is,

$$
x^{*}=P_{\Omega}(I-(A-\gamma f)) x^{*}
$$

Proof. Since $\epsilon_{n} \rightarrow 0$ as $n \rightarrow \infty$ by the condition (E1), we may assume, without loss of generality, that $\epsilon_{n}<\left(1-\delta_{n}\|B\|\right)\|A\|^{-1}$ for all $n \geq 1$. Noting that $A$ and $B$ are both the linear bounded self-adjoint operators, one has

$$
\begin{gathered}
\|A\|=\sup \{|\langle A x, x\rangle|: x \in H,\|x\|=1\}, \\
\|B\|=\sup \{|\langle B x, x\rangle|: x \in H,\|x\|=1\} .
\end{gathered}
$$

Observing that

$$
\begin{aligned}
\left\langle\left(I-\delta_{n} B-\epsilon_{n} A\right) x, x\right\rangle & =1-\delta_{n}\langle B x, x\rangle-\epsilon_{n}\langle A x, x\rangle \\
& \geq 1-\delta_{n}\|B\|-\epsilon_{n}\|A\| \\
& \geq 0,
\end{aligned}
$$

we obtain that $I-\delta_{n} B-\epsilon_{n} A$ is positive for all $n \geq 1$. It follows that

$$
\begin{aligned}
\left\|I-\delta_{n} B-\epsilon_{n} A\right\| & =\sup \left\{\left\langle\left(I-\delta_{n} B-\epsilon_{n} A\right) x, x\right\rangle: x \in H,\|x\|=1\right\} \\
& =\sup \left\{1-\left\langle\left(\delta_{n} B+\epsilon_{n} A\right) x, x\right\rangle: x \in H,\|x\|=1\right\} \\
& \leq 1-\delta_{n} \bar{\beta}-\epsilon_{n} \bar{\gamma} .
\end{aligned}
$$

For each $n \geq 1$, define a quadratic function $f\left(\delta_{n}\right)$ in $\delta_{n}$ as follows:

$$
f\left(\delta_{n}\right)=2 \bar{\beta}\|B\| \delta_{n}^{2}+\left(\bar{\beta}-\|B\|^{2}-2\|B\|\right) \delta_{n}
$$

Note that

$$
\begin{gathered}
f(0)=f\left(\frac{2\|B\|-\bar{\beta}+\|B\|^{2}}{2 \bar{\beta}\|B\|}\right)=0 \\
f\left(\frac{2\|B\|+\|B\|^{2}-\bar{\beta}+\sqrt{\left(\bar{\beta}-\|B\|^{2}-2\|B\|\right)^{2}+8 \bar{\beta}\|B\|}}{4 \bar{\beta}\|B\|}\right)=1 .
\end{gathered}
$$

Hence, for each $\delta_{n}$ satisfying the condition (E4), one has

$$
0<2 \bar{\beta}\|B\| \delta_{n}^{2}+\left(\bar{\beta}-\|B\|^{2}-2\|B\|\right) \delta_{n}<1 .
$$


Moreover, it follows from (3.7), $f(1 /\|B\|)>1$ and (E4) that

$$
\delta_{n}<\frac{1}{\|B\|}, \quad \forall n \geq 1
$$

Next, we proceed the proof with following steps.

Step 1. $\left\{x_{n}\right\}$ is bounded.

Let $p \in \Omega$. Lemma 2.6 shows that every $T_{r_{i}}$ is firmly nonexpansive and hence nonexpansive. Since $r<r_{i}<2 k_{i}, I-r_{i} A_{i}$ is nonexpansive for each $i \geq 1$. Therefore, $T_{r_{i}}\left(I-r_{i} A_{i}\right)$ is nonexpansive for each $i \geq 1$. Noting that $\left\{\alpha_{n}\right\}$ is strictly decreasing, $\alpha_{0}=1$, we have

$$
\begin{aligned}
\left\|z_{n}-p\right\| & =\left\|\alpha_{n}\left(x_{n}-p\right)+\sum_{i=1}^{n}\left(\alpha_{i-1}-\alpha_{i}\right)\left(T_{r_{i}}\left(I-r_{i} A_{i}\right) x_{n}-T_{r_{i}}\left(I-r_{i} A_{i}\right) p\right)\right\| \\
& \leq \alpha_{n}\left\|x_{n}-p\right\|+\sum_{i=1}^{n}\left(\alpha_{i-1}-\alpha_{i}\right)\left\|T_{r_{i}}\left(I-r_{i} A_{i}\right) x_{n}-T_{r_{i}}\left(I-r_{i} A_{i}\right) p\right\| \\
& \leq \alpha_{n}\left\|x_{n}-p\right\|+\sum_{i=1}^{n}\left(\alpha_{i-1}-\alpha_{i}\right)\left\|x_{n}-p\right\| \\
& =\left\|x_{n}-p\right\|
\end{aligned}
$$

and hence

$$
\left\|W_{n} z_{n}-p\right\|=\left\|W_{n} z_{n}-W_{n} p\right\| \leq\left\|z_{n}-p\right\| \leq\left\|x_{n}-p\right\| .
$$

Then, from (3.4) and (3.11), it follows that (noting that $B$ is linear and $\bar{\beta}>2\|B\|+\|B\|^{2} \Rightarrow \bar{\beta}>$ $\|B\|)$

$$
\begin{aligned}
\| x_{n+1} & -p \| \\
& =\left\|\epsilon_{n}\left(\gamma f\left(x_{n}\right)-A p\right)+\delta_{n}\left(B x_{n}-B p\right)+\left(I-\delta_{n} B-\epsilon_{n} A\right)\left(W_{n} z_{n}-p\right)\right\| \\
& \leq \epsilon_{n}\left\|\gamma f\left(x_{n}\right)-A p\right\|+\delta_{n}\left\|B\left(x_{n}-p\right)\right\|+\left\|I-\delta_{n} B-\epsilon_{n} A\right\|\left\|W_{n} z_{n}-p\right\| \\
& \leq \epsilon_{n} \gamma \kappa\left\|x_{n}-p\right\|+\epsilon_{n}\|\gamma f(p)-A p\|+\delta_{n}\|B\|\left\|x_{n}-p\right\|+\left(1-\delta_{n} \bar{\beta}-\epsilon_{n} \bar{\gamma}\right)\left\|W_{n} z_{n}-p\right\| \\
& \leq \epsilon_{n} \gamma \kappa\left\|x_{n}-p\right\|+\epsilon_{n}\|\gamma f(p)-A p\|+\delta_{n}\|B\|\left\|x_{n}-p\right\|+\left(1-\delta_{n} \bar{\beta}-\epsilon_{n} \bar{\gamma}\right)\left\|x_{n}-p\right\| \\
& \leq\left(1-\epsilon_{n}(\bar{\gamma}-\gamma \mathcal{K})\right)\left\|x_{n}-p\right\|+\epsilon_{n}\|\gamma f(p)-A p\| .
\end{aligned}
$$


It follows from $\epsilon_{n} \in(0,1)$ and $0<\bar{\gamma}-\gamma \kappa<1$ that $0<\epsilon_{n}(\bar{\gamma}-\gamma \kappa)<1$. Therefore, by the simple induction, we have

$$
\left\|x_{n}-p\right\| \leq \max \left\{\left\|x_{1}-p\right\|, \frac{\|\gamma f(p)-A p\|}{\bar{\gamma}-\gamma \kappa}\right\}, \quad \forall n \geq 1
$$

which shows that $\left\{x_{n}\right\}$ is bounded, so is $\left\{z_{n}\right\}$.

Step 2. $\left\|x_{n+1}-x_{n}\right\| \rightarrow 0$ as $n \rightarrow \infty$.

First, we prove

$$
\lim _{n \rightarrow \infty}\left\|W_{n+1} z_{n}-W_{n} z_{n}\right\|=0
$$

Let $i \in\{0,1, \ldots, N-2\}$ and set

$$
M_{1}=\sup _{n}\left\{\left\|z_{n}\right\|+\left\|T_{1} z_{n}\right\|+\sum_{i=2}^{N}\left\|T_{i} U_{n, i-1} z_{n}\right\|\right\}<\infty .
$$

It follows from the definition of $W_{n}$ that

$$
\begin{aligned}
&\left\|U_{n+1, N-i} z_{n}-U_{n, N-i} z_{n}\right\| \\
&=\left\|\lambda_{n+1, N-i} T_{N-i} U_{n+1, N-i-1} z_{n}+\left(1-\lambda_{n+1, N-i}\right) z_{n}-\lambda_{n, N-i} T_{N-i} U_{n, N-i-1} z_{n}-\left(1-\lambda_{n, N-i}\right) z_{n}\right\| \\
& \leq \lambda_{n+1, N-i}\left\|T_{N-i} U_{n+1, N-i-1} z_{n}-T_{N-i} U_{n, N-i-1} z_{n}\right\| \\
&+\left|\lambda_{n+1, N-i}-\lambda_{n, N-i}\right|\left\|T_{N-i} U_{n, N-i-1} z_{n}\right\|+\left|\lambda_{n+1, N-i}-\lambda_{n, N-i}\right|\left\|z_{n}\right\| \\
& \leq\left\|U_{n+1, N-i-1} z_{n}-U_{n, N-i-1} z_{n}\right\|+\left(\left\|z_{n}\right\|+\left\|T_{N-i} U_{n, N-i-1} z_{n}\right\|\right)\left|\lambda_{n+1, N-i}-\lambda_{n, N-i}\right| \\
& \leq\left\|U_{n+1, N-i-1} z_{n}-U_{n, N-i-1} z_{n}\right\|+M_{1}\left|\lambda_{n+1, N-i}-\lambda_{n, N-i}\right|
\end{aligned}
$$

for each $i \in\{0,1, \ldots, N-2\}$. Thus, using the above recursive inequalities repeatedly, we have

$$
\begin{aligned}
\left\|W_{n+1} z_{n}-W_{n} z_{n}\right\| & =\left\|U_{n+1, N} z_{n}-U_{n, N} z_{n}\right\| \\
& \leq M_{1} \sum_{i=2}^{N}\left|\lambda_{n+1, i}-\lambda_{n, i}\right|+\left|\lambda_{n+1,1}-\lambda_{n, 1}\right|\left(\left\|z_{n}\right\|+\left\|T_{1} z_{n}\right\|\right) \\
& \leq M_{1} \sum_{i=1}^{N}\left|\lambda_{n+1, i}-\lambda_{n, i}\right| .
\end{aligned}
$$


Also, we have

$$
\begin{aligned}
\left\|z_{n}-z_{n-1}\right\|= & \| \alpha_{n} x_{n}+\sum_{i=1}^{n}\left(\alpha_{i-1}-\alpha_{i}\right) T_{r_{i}}\left(I-r_{i} A_{i}\right) x_{n}-\alpha_{n-1} x_{n-1} \\
& \quad-\sum_{i=1}^{n}\left(\alpha_{i-1}-\alpha_{i}\right) T_{r_{i}}\left(I-r_{i} A_{i}\right) x_{n-1}+\left(\alpha_{n-1}-\alpha_{n}\right) T_{r_{n}}\left(I-r_{n} A_{n}\right) x_{n-1} \| \\
\leq & \alpha_{n}\left\|x_{n}-x_{n-1}\right\|+\left|\alpha_{n}-\alpha_{n-1}\right|\left\|x_{n-1}\right\| \\
& +\sum_{i=1}^{n}\left(\alpha_{i-1}-\alpha_{i}\right)\left\|x_{n}-x_{n-1}\right\|+\left|\alpha_{n-1}-\alpha_{n}\right|\left\|T_{r_{n}}\left(I-r_{n} A_{n}\right) x_{n-1}\right\| \\
= & \left\|x_{n}-x_{n-1}\right\|+\left|\alpha_{n}-\alpha_{n-1}\right|\left\|x_{n-1}\right\|+\left|\alpha_{n-1}-\alpha_{n}\right|\left\|T_{r_{n}}\left(I-r_{n} A_{n}\right) x_{n-1}\right\| \\
\leq & \left\|x_{n}-x_{n-1}\right\|+\left|\alpha_{n}-\alpha_{n-1}\right| L,
\end{aligned}
$$

where $L=\sup \left\{\left\|x_{n-1}\right\|+\left\|T_{r_{n}}\left(I-r_{n} A_{n}\right) x_{n-1}\right\|\right\}$.

Next, we prove $\lim _{n \rightarrow \infty}\left\|x_{n+1}-x_{n}\right\|=0$. Observe (noting that $B$ is linear) that

$$
\begin{aligned}
x_{n+1}-x_{n}= & \epsilon_{n} \gamma\left(f\left(x_{n}\right)-f\left(x_{n-1}\right)\right)+\epsilon_{n} \gamma f\left(x_{n-1}\right)+\delta_{n} B\left(x_{n}-x_{n-1}\right)+\delta_{n} B x_{n-1} \\
& +\left(I-\delta_{n} B-\epsilon_{n} A\right)\left(W_{n} z_{n}-W_{n} z_{n-1}\right)+\left(I-\delta_{n} B-\epsilon_{n} A\right) W_{n} z_{n-1} \\
& -\epsilon_{n-1} \gamma f\left(x_{n-1}\right)-\delta_{n-1} B x_{n-1}-\left(I-\delta_{n-1} B-\epsilon_{n-1} A\right) W_{n-1} z_{n-1} \\
= & \epsilon_{n} \gamma\left(f\left(x_{n}\right)-f\left(x_{n-1}\right)\right)+\delta_{n} B\left(x_{n}-x_{n-1}\right)+\left(I-\delta_{n} B-\epsilon_{n} A\right)\left(W_{n} z_{n}-W_{n} z_{n-1}\right) \\
& +\left(\epsilon_{n}-\epsilon_{n-1}\right) \gamma f\left(x_{n-1}\right)+\left(\delta_{n}-\delta_{n-1}\right) B x_{n-1}+\left(W_{n} z_{n-1}-W_{n-1} z_{n-1}\right) \\
& +\left(\delta_{n-1} B W_{n-1} z_{n-1}-\delta_{n} B W_{n} z_{n-1}\right)+\left(\epsilon_{n-1} A W_{n-1} z_{n-1}-\epsilon_{n} A W_{n} z_{n-1}\right) \\
= & \epsilon_{n} \gamma\left(f\left(x_{n}\right)-f\left(x_{n-1}\right)\right)+\delta_{n} B\left(x_{n}-x_{n-1}\right)+\left(I-\delta_{n} B-\epsilon_{n} A\right)\left(W_{n} z_{n}-W_{n} z_{n-1}\right) \\
& +\left(\epsilon_{n}-\epsilon_{n-1}\right) \gamma f\left(x_{n-1}\right)+\left(\delta_{n}-\delta_{n-1}\right) B x_{n-1}+\left(W_{n} z_{n-1}-W_{n-1} z_{n-1}\right) \\
& +\left(\delta_{n-1}-\delta_{n}\right) B W_{n-1} z_{n-1}+\delta_{n} B\left(W_{n-1} z_{n-1}-W_{n} z_{n-1}\right) \\
& +\left(\epsilon_{n-1}-\epsilon_{n}\right) A W_{n-1} z_{n-1}+\epsilon_{n} A\left(W_{n-1} z_{n-1}-W_{n} z_{n-1}\right) .
\end{aligned}
$$


Hence, by (3.4) and (3.18), we get

$$
\begin{aligned}
\left\|x_{n+1}-x_{n}\right\| \leq & \epsilon_{n} \gamma \kappa\left\|x_{n}-x_{n-1}\right\|+\delta_{n}\|B\|\left\|x_{n}-x_{n-1}\right\|+\left(1-\delta_{n} \bar{\beta}-\epsilon_{n} \bar{\gamma}\right)\left\|z_{n}-z_{n-1}\right\| \\
& +\left|\epsilon_{n}-\epsilon_{n-1}\right| \gamma\left\|f\left(x_{n-1}\right)\right\|+\left|\delta_{n}-\delta_{n-1}\right|\left\|B x_{n-1}\right\|+\left\|W_{n} z_{n-1}-W_{n-1} z_{n-1}\right\| \\
& +\left|\delta_{n-1}-\delta_{n}\right|\left\|B W_{n-1} z_{n-1}\right\|+\delta_{n}\|B\|\left\|W_{n-1} z_{n-1}-W_{n} z_{n-1}\right\| \\
& +\left|\epsilon_{n-1}-\epsilon_{n}\right|\left\|A W_{n-1} z_{n-1}\right\|+\epsilon_{n}\|A\|\left\|W_{n-1} z_{n-1}-W_{n} z_{n-1}\right\| \\
\leq & \epsilon_{n} \gamma \kappa\left\|x_{n}-x_{n-1}\right\|+\delta_{n}\|B\|\left\|x_{n}-x_{n-1}\right\| \\
& +\left(1-\delta_{n} \bar{\beta}-\epsilon_{n} \bar{\gamma}\right)\left(\left\|x_{n}-x_{n-1}\right\|+\left|\alpha_{n}-\alpha_{n-1}\right| L\right) \\
& +\left|\epsilon_{n}-\epsilon_{n-1}\right| \gamma\left\|f\left(x_{n-1}\right)\right\|+\left|\delta_{n}-\delta_{n-1}\right|\left\|B x_{n-1}\right\|+\left\|W_{n} z_{n-1}-W_{n-1} z_{n-1}\right\| \\
& +\left|\delta_{n-1}-\delta_{n}\right|\left\|B W_{n-1} z_{n-1}\right\|+\delta_{n}\|B\|\left\|W_{n-1} z_{n-1}-W_{n} z_{n-1}\right\| \\
& +\left|\epsilon_{n-1}-\epsilon_{n}\right|\left\|A W_{n-1} z_{n-1}\right\|+\epsilon_{n}\|A\|\left\|W_{n-1} z_{n-1}-W_{n} z_{n-1}\right\| \\
\leq & {\left[1-\left(\delta_{n}(\bar{\beta}-\|B\|)+\epsilon_{n}(\bar{\gamma}-\gamma \kappa)\right)\right]\left\|x_{n}-x_{n-1}\right\|+L\left|\alpha_{n}-\alpha_{n-1}\right|+2\left|\epsilon_{n-1}-\epsilon_{n}\right| M_{2} } \\
& +2\left|\delta_{n-1}-\delta_{n}\right| M_{2}+\left(1+\delta_{n}\|B\|+\epsilon_{n}\|A\|\right)\left\|W_{n-1} z_{n-1}-W_{n} z_{n-1}\right\|,
\end{aligned}
$$

where $M_{2}=\sup _{n}\left\{\gamma\left\|f\left(x_{n-1}\right)\right\|+\left\|B x_{n-1}\right\|+\left\|B W_{n-1} z_{n-1}\right\|+\left\|A W_{n-1} z_{n-1}\right\|\right\}$.

Set $M_{3}=\min \{\bar{\beta}-\|B\|, \bar{\gamma}-\gamma \kappa\}$. It follows from $0 \leq \bar{\gamma}-\gamma \kappa<1$ and $\bar{\beta}>\|B\|$ (due to $\bar{\beta}>2\|B\|+\|B\|^{2}$ ) that $0 \leq M_{2}<1$. Thus we have

$$
\begin{aligned}
& \left\|x_{n+1}-x_{n}\right\| \leq\left[1-\left(\delta_{n}+\epsilon_{n}\right) M_{3}\right]\left\|x_{n}-x_{n-1}\right\|+\left(\delta_{n}+\epsilon_{n}\right) M_{3} \\
& \times\left(\frac{1}{\left(\delta_{n}+\epsilon_{n}\right) M_{3}}+\frac{\delta_{n}\|B\|}{\left(\delta_{n}+\epsilon_{n}\right) M_{3}}+\frac{\epsilon_{n}\|A\|}{\left(\delta_{n}+\epsilon_{n}\right) M_{3}}\right) \\
& \times|| W_{n-1} z_{n-1}-W_{n} z_{n-1} \|+L\left|\alpha_{n}-\alpha_{n-1}\right|+2\left|\epsilon_{n-1}-\epsilon_{n}\right| M_{2}+2\left|\delta_{n-1}-\delta_{n}\right| M_{2} .
\end{aligned}
$$

Set

$$
\begin{gathered}
\eta_{n}=\left(\delta_{n}+\epsilon_{n}\right) M_{3}, \\
\tau_{n}=\left(\frac{1}{\left(\delta_{n}+\epsilon_{n}\right) M_{3}}+\frac{\delta_{n}\|B\|}{\left(\delta_{n}+\epsilon_{n}\right) M_{3}}+\frac{\epsilon_{n}\|A\|}{\left(\delta_{n}+\epsilon_{n}\right) M_{3}}\right)\left\|W_{n-1} z_{n-1}-W_{n} z_{n-1}\right\|, \\
\xi_{n}=L\left|\alpha_{n}-\alpha_{n-1}\right|+2\left|\epsilon_{n-1}-\epsilon_{n}\right| M_{2}+2\left|\delta_{n-1}-\delta_{n}\right| M_{2} .
\end{gathered}
$$


Fixed Point Theory and Applications

Then it follows from (3.21) that

$$
\left\|x_{n+1}-x_{n}\right\| \leq\left(1-\eta_{n}\right)\left\|x_{n}-x_{n-1}\right\|+\eta_{n} \tau_{n}+\xi_{n} .
$$

It follows from the assumption condition (E1), (E3), (E5), and (3.14) that

$$
\eta_{n} \subset[0,1], \quad \sum_{n=1}^{\infty} \eta_{n}=\infty, \quad \lim _{n \rightarrow \infty} \tau_{n}=0, \quad \sum_{n=1}^{\infty} \xi_{n}<\infty
$$

By applying Lemma 2.2 to (3.23), we obtain $\left\|x_{n+1}-x_{n}\right\| \rightarrow 0$ as $n \rightarrow \infty$.

Step 3. $x_{n}-W_{n} z_{n} \rightarrow 0$ as $n \rightarrow \infty$.

For all $n \geq 1$, we have

$$
\begin{aligned}
\left\|x_{n}-W_{n} z_{n}\right\| & \leq\left\|x_{n}-x_{n+1}\right\|+\left\|x_{n+1}-W_{n} z_{n}\right\| \\
& =\left\|x_{n}-x_{n+1}\right\|+\left\|\epsilon_{n} \gamma f\left(x_{n}\right)+\delta_{n} B x_{n}+\left[I-\delta_{n} B-\epsilon_{n} A\right] W_{n} z_{n}-W_{n} z_{n}\right\| \\
& \leq\left\|x_{n}-x_{n+1}\right\|+\epsilon_{n}\left\|\gamma f\left(x_{n}\right)-A W_{n} z_{n}\right\|+\delta_{n}\left\|B x_{n}-B W_{n} z_{n}\right\| \\
& \leq\left\|x_{n}-x_{n+1}\right\|+\epsilon_{n}\left\|\gamma f\left(x_{n}\right)-A W_{n} z_{n}\right\|+\delta_{n}\|B\|\left\|x_{n}-W_{n} z_{n}\right\|
\end{aligned}
$$

and hence (noting (3.9))

$$
\left\|x_{n}-W_{n} z_{n}\right\| \leq \frac{1}{1-\delta_{n}\|B\|}\left\|x_{n}-x_{n+1}\right\|+\frac{\epsilon_{n}}{1-\delta_{n}}\left[\gamma\left\|f\left(x_{n}\right)\right\|+\left\|A W_{n} z_{n}\right\|\right] .
$$

It follows from the assumption conditions (E1), (E2), and Step 2 that

$$
x_{n}-W_{n} z_{n} \longrightarrow 0 \quad(n \longrightarrow \infty)
$$

Step 4. $x_{n}-z_{n} \rightarrow 0$ as $n \rightarrow \infty$. 
Notice that, for any $x \in \Omega$,

$$
\begin{aligned}
\left\|z_{n}-x\right\|^{2} & =\left\|\alpha_{n}\left(x_{n}-x\right)+\sum_{i=1}^{n}\left(\alpha_{i-1}-\alpha_{i}\right)\left(T_{r_{i}}\left(I-r_{i} A_{i}\right) x_{n}-T_{r_{i}}\left(I-r_{i} A_{i}\right) x\right)\right\|^{2} \\
& \leq \alpha_{n}\left\|x_{n}-x\right\|^{2}+\sum_{i=1}^{n}\left(\alpha_{i-1}-\alpha_{i}\right)\left\|\left(I-r_{i} A_{i}\right) x_{n}-\left(I-r_{i} A_{i}\right) x\right\|^{2} \\
& \leq \alpha_{n}\left\|x_{n}-x\right\|^{2}+\sum_{i=1}^{n}\left(\alpha_{i-1}-\alpha_{i}\right)\left[\left\|x_{n}-x\right\|^{2}+r_{i}\left(r_{i}-2 k_{i}\right)\left\|A_{i} x_{n}-A_{i} x\right\|^{2}\right] \\
& =\left\|x_{n}-x\right\|^{2}+\sum_{i=1}^{n}\left(\alpha_{i-1}-\alpha_{i}\right) r_{i}\left(r_{i}-2 k_{i}\right)\left\|A_{i} x_{n}-A_{i} x\right\|^{2} .
\end{aligned}
$$

Let $y_{n}=\gamma f\left(x_{n}\right)-A W_{n} z_{n}$ and $\lambda=\sup \left\{\left\|\gamma f\left(x_{n}\right)-A W_{n} z_{n}\right\|: n \geq 1\right\}$. By using (3.8), (3.9), (3.28), Lemmas 2.3, and 2.4, we have (noting that $\delta_{n}<1 / \bar{\beta}$ )

$$
\begin{aligned}
\left\|x_{n+1}-x\right\|^{2}= & \left\|\left(I-\delta_{n} B\right)\left(W_{n} z_{n}-x\right)+\delta_{n}\left(B x_{n}-B x\right)+\epsilon_{n}\left(\gamma f\left(x_{n}\right)-A W_{n} z_{n}\right)\right\|^{2} \\
\leq & \left\|\left(I-\delta_{n} B\right)\left(W_{n} z_{n}-x\right)+\delta_{n}\left(B x_{n}-B x\right)\right\|^{2}+2 \epsilon_{n}\left\langle y_{n}, x_{n+1}-x\right\rangle \\
= & \left\|\left(I-\delta_{n} B\right)\left(W_{n} z_{n}-W_{n} x\right)+\delta_{n} B\left(x_{n}-x\right)\right\|^{2}+2 \epsilon_{n}\left\langle y_{n}, x_{n+1}-x\right\rangle \\
\leq & \left(1-\delta_{n} \bar{\beta}\right)\left\|z_{n}-x\right\|^{2}+\delta_{n}\|B\|^{2}\left\|x_{n}-x\right\|^{2}+2 \delta_{n}\left(1-\delta_{n} \bar{\beta}\right)\|B\|\left\|x_{n}-x\right\|^{2}+2 \lambda^{2} \epsilon_{n} \\
\leq & \left(1-\delta_{n} \bar{\beta}\right)\left[\left\|x_{n}-x\right\|^{2}+\sum_{i=1}^{n}\left(\alpha_{i-1}-\alpha_{i}\right) r_{i}\left(r_{i}-2 k_{i}\right)\left\|A_{i} x_{n}-A_{i} x\right\|^{2}\right] \\
& +\delta_{n}\|B\|^{2}\left\|x_{n}-x\right\|^{2}+2 \delta_{n}\left(1-\delta_{n} \bar{\beta}\right)\|B\|\left\|x_{n}-x\right\|^{2}+2 \lambda^{2} \epsilon_{n} \\
= & {\left[1-\left(\delta_{n} \bar{\beta}-\delta_{n}\|B\|^{2}-2 \delta_{n}\left(1-\delta_{n} \bar{\beta}\right)\|B\|\right)\right]\left\|x_{n}-x\right\|^{2} } \\
& +\left(1-\delta_{n} \bar{\beta}\right) \sum_{i=1}^{n}\left(\alpha_{i-1}-\alpha_{i}\right) r_{i}\left(r_{i}-2 k_{i}\right)\left\|A_{i} x_{n}-A_{i} x\right\|^{2}+2 \lambda^{2} \epsilon_{n} \\
\leq & \left\|x_{n}-x\right\|^{2}+\left(1-\delta_{n} \bar{\beta}\right) \sum_{i=1}^{n}\left(\alpha_{i-1}-\alpha_{i}\right) r_{i}\left(r_{i}-2 k_{i}\right)\left\|A_{i} x_{n}-A_{i} x\right\|^{2}+2 \lambda^{2} \epsilon_{n} .
\end{aligned}
$$

This shows that

$$
\begin{aligned}
& \left(1-\delta_{n} \bar{\beta}\right) \sum_{i=1}^{n}\left(\alpha_{i-1}-\alpha_{i}\right) r_{i}\left(2 k_{i}-r_{i}\right)\left\|A_{i} x_{n}-A_{i} x\right\|^{2} \\
& \leq\left\|x_{n}-x\right\|^{2}-\left\|x_{n+1}-x\right\|^{2}+2 \lambda^{2} \epsilon_{n} \\
& \leq\left(\left\|x_{n}-x\right\|+\left\|x_{n+1}-x\right\|\right)\left\|x_{n}-x_{n+1}\right\|+2 \lambda^{2} \epsilon_{n}
\end{aligned}
$$


and hence, for each $i \geq 1$,

$$
\begin{aligned}
& \left(1-\delta_{n} \bar{\beta}\right)\left(\alpha_{i-1}-\alpha_{i}\right) r_{i}\left(2 k_{i}-r_{i}\right)\left\|A_{i} x_{n}-A_{i} x\right\|^{2} \\
& \quad \leq\left\|x_{n}-x\right\|^{2}-\left\|x_{n+1}-x\right\|^{2}+2 \lambda^{2} \epsilon_{n} \\
& \quad \leq\left(\left\|x_{n}-x\right\|+\left\|x_{n+1}-x\right\|\right)\left\|x_{n}-x_{n+1}\right\|+2 \lambda^{2} \epsilon_{n} .
\end{aligned}
$$

Since $\delta_{n} \rightarrow 0,\left\|x_{n}-x_{n+1}\right\| \rightarrow 0$ and $\alpha_{i-1}-\alpha_{i}>0$, we have

$$
\lim _{n \rightarrow \infty}\left\|A_{i} x_{n}-A_{i} x\right\|=0, \quad i \geq 1 .
$$

Now, for $x \in \Omega$, we have, from Lemma 2.2,

$$
\begin{aligned}
\| T_{r_{i}}(I- & \left.r_{i} A_{i}\right) x_{n}-x \|^{2} \\
= & \left\|T_{r_{i}}\left(I-r_{i} A_{i}\right) x_{n}-T_{r_{i}}\left(I-r_{i} A_{i}\right) x\right\|^{2} \\
\leq & \left\langle T_{r_{i}}\left(I-r_{i} A_{i}\right) x_{n}-T_{r_{i}}\left(I-r_{i} A_{i}\right) x,\left(I-r_{i} A_{i}\right) x_{n}-\left(I-r_{i} A_{i}\right) x\right\rangle \\
= & \left\langle T_{r_{i}}\left(I-r_{i} A_{i}\right) x_{n}-x, x_{n}-x\right\rangle+r_{i}\left\langle T_{r_{i}}\left(I-r_{i} A_{i}\right) x_{n}-x, A_{i} x-A_{i} x_{n}\right\rangle \\
= & \frac{1}{2}\left\{\left\|T_{r_{i}}\left(I-r_{i} A_{i}\right) x_{n}-x\right\|^{2}+\left\|x_{n}-x\right\|^{2}-\left\|x_{n}-T_{r_{i}}\left(I-r_{i} A_{i}\right) x_{n}\right\|^{2}\right\} \\
& +r_{i}\left\langle T_{r_{i}}\left(I-r_{i} A_{i}\right) x_{n}-x, A_{i} x-A_{i} x_{n}\right\rangle
\end{aligned}
$$

and hence

$$
\begin{aligned}
\left\|T_{r_{i}}\left(I-r_{i} A_{i}\right) x_{n}-x\right\|^{2} \leq & \left\|x_{n}-x\right\|^{2}-\left\|x_{n}-T_{r_{i}}\left(I-r_{i} A_{i}\right) x_{n}\right\|^{2} \\
& +2 r_{i}\left\langle T_{r_{i}}\left(I-r_{i} A_{i}\right) x_{n}-x, A_{i} x-A_{i} x_{n}\right\rangle .
\end{aligned}
$$

Therefore,

$$
\begin{aligned}
\left\|z_{n}-x\right\|^{2} \leq & \alpha_{n}\left\|x_{n}-x\right\|^{2}+\sum_{i=1}^{n}\left(\alpha_{i-1}-\alpha_{i}\right)\left\|T_{r_{i}}\left(I-r_{i} A_{i}\right) x_{n}-x\right\|^{2} \\
\leq & \alpha_{n}\left\|x_{n}-x\right\|^{2}+\sum_{i=1}^{n}\left(\alpha_{i-1}-\alpha_{i}\right)\left[\left\|x_{n}-x\right\|^{2}-\left\|x_{n}-T_{r_{i}}\left(I-r_{i} A_{i}\right) x_{n}\right\|^{2}\right. \\
& \left.+2 r_{i}\left\langle T_{r_{i}}\left(I-r_{i} A_{i}\right) x_{n}-x, A_{i} x-A_{i} x_{n}\right\rangle\right] \\
= & \left\|x_{n}-x\right\|^{2}-\sum_{i=1}^{n}\left(\alpha_{i-1}-\alpha_{i}\right)\left\|x_{n}-T_{r_{i}}\left(I-r_{i} A_{i}\right) x_{n}\right\|^{2} \\
& +2 \sum_{i=1}^{n}\left(\alpha_{i-1}-\alpha_{i}\right) r_{i}\left\langle T_{r_{i}}\left(I-r_{i} A_{i}\right) x_{n}-x, A_{i} x-A_{i} x_{n}\right\rangle .
\end{aligned}
$$


By using (3.8), (3.9), (3.35), Lemmas 2.3 and 2.4, we have (noting that $\delta_{n}<1 / \bar{\beta}$ )

$$
\begin{aligned}
\| x_{n+1}- & x \|^{2} \\
= & \left\|\left(I-\delta_{n} B\right)\left(W_{n} z_{n}-x\right)+\delta_{n}\left(B x_{n}-B x\right)+\epsilon_{n}\left(\gamma f\left(x_{n}\right)-A W_{n} z_{n}\right)\right\|^{2} \\
\leq & \left\|\left(I-\delta_{n} B\right)\left(W_{n} z_{n}-x\right)+\delta_{n}\left(B x_{n}-B x\right)\right\|^{2}+2 \epsilon_{n}\left\langle y_{n}, x_{n+1}-x\right\rangle \\
= & \left\|\left(I-\delta_{n} B\right)\left(W_{n} z_{n}-W_{n} x\right)+\delta_{n} B\left(x_{n}-x\right)\right\|^{2}+2 \epsilon_{n}\left\langle y_{n}, x_{n+1}-x\right\rangle \\
\leq & \left(1-\delta_{n} \bar{\beta}\right)\left\|z_{n}-x\right\|^{2}+\delta_{n}\|B\|^{2}\left\|x_{n}-x\right\|^{2}+2 \delta_{n}\left(1-\delta_{n} \bar{\beta}\right)\|B\|\left\|x_{n}-x\right\|^{2}+2 \lambda^{2} \epsilon_{n} \\
\leq & \left(1-\delta_{n} \bar{\beta}\right)\left[\left\|x_{n}-x\right\|^{2}-\sum_{i=1}^{n}\left(\alpha_{i-1}-\alpha_{i}\right)\left\|x_{n}-T_{r_{i}}\left(I-r_{i} A_{i}\right) x_{n}\right\|^{2}\right. \\
& \left.+2 \sum_{i=1}^{n}\left(\alpha_{i-1}-\alpha_{i}\right) r_{i}\left\langle T_{r_{i}}\left(I-r_{i} A_{i}\right) x_{n}-x, A_{i} x-A_{i} x_{n}\right\rangle\right] \\
& +\delta_{n}\|B\|^{2}\left\|x_{n}-x\right\|^{2}+2 \delta_{n}\left(1-\delta_{n} \bar{\beta}\right)\|B\|\left\|x_{n}-x\right\|^{2}+2 \lambda^{2} \epsilon_{n} \\
= & {\left[1-\left(\delta_{n} \bar{\beta}-\delta_{n}\|B\|^{2}-2 \delta_{n}\left(1-\delta_{n} \bar{\beta}\right)\|B\|\right)\right]\left\|x_{n}-x\right\|^{2} } \\
& -\left(1-\delta_{n} \bar{\beta}\right) \sum_{i=1}^{n}\left(\alpha_{i-1}-\alpha_{i}\right)\left\|x_{n}-T_{r_{i}}\left(I-r_{i} A_{i}\right) x_{n}\right\|^{2} \\
\leq & \left\|x_{n}-x\right\|^{2}-\left(1-\delta_{n} \bar{\beta}\right) \sum_{i=1}^{n}\left(\alpha_{i-1}-\alpha_{i}\right)\left\|x_{n}-T_{r_{i}}\left(I-r_{i} A_{i}\right) x_{n}\right\|^{2} \\
& +2\left(1-\delta_{n} \bar{\beta}\right) \sum_{i=1}^{n}\left(\alpha_{i-1}-\alpha_{i}\right) r_{i}\left\langle T_{r_{i}}\left(I-r_{i} A_{i}\right) x_{n}-x, \alpha_{i} x-A_{i} x_{n}\right\rangle+2 \lambda^{2} \epsilon_{n} \\
& +2\left(\alpha_{r_{i}}\left(I-r_{i} A_{i}\right) x_{n}-x, A_{i} x-A_{i} x_{n}\right\rangle+2 \lambda^{2} \epsilon_{n} \\
& \\
& \\
& \\
&
\end{aligned}
$$

and hence

$$
\begin{aligned}
& \left(1-\delta_{n} \bar{\beta}\right) \sum_{i=1}^{n}\left(\alpha_{i-1}-\alpha_{i}\right)\left\|x_{n}-T_{r_{i}}\left(I-r_{i} A_{i}\right) x_{n}\right\|^{2} \\
& \leq\left\|x_{n}-x\right\|^{2}-\left\|x_{n+1}-p\right\|^{2}+2\left(1-\delta_{n} \bar{\beta}\right) \\
& \quad \times \sum_{i=1}^{n}\left(\alpha_{i-1}-\alpha_{i}\right) r_{i}\left\langle T_{r_{i}}\left(I-r_{i} A_{i}\right) x_{n}-x, A_{i} x-A_{i} x_{n}\right\rangle+2 \lambda^{2} \epsilon_{n} \\
& \leq\left(\left\|x_{n}-x\right\|+\left\|x_{n+1}-x\right\|\right)\left\|x_{n}-x_{n+1}\right\| \\
& \quad+2\left(1-\delta_{n} \bar{\beta}\right) \sum_{i=1}^{n}\left(\alpha_{i-1}-\alpha_{i}\right) r_{i}\left\langle T_{r_{i}}\left(I-r_{i} A_{i}\right) x_{n}-x, A_{i} x-A_{i} x_{n}\right\rangle+2 \lambda^{2} \epsilon_{n} .
\end{aligned}
$$


This shows that for, each $i \geq 1$,

$$
\begin{aligned}
& \left(1-\delta_{n} \bar{\beta}\right)\left(\alpha_{i-1}-\alpha_{i}\right)\left\|x_{n}-T_{r_{i}}\left(I-r_{i} A_{i}\right) x_{n}\right\|^{2} \\
& \quad \leq\left(\left\|x_{n}-x\right\|+\left\|x_{n+1}-x\right\|\right)\left\|x_{n}-x_{n+1}\right\| \\
& \quad+2\left(1-\delta_{n} \bar{\beta}\right) \sum_{i=1}^{n}\left(\alpha_{i-1}-\alpha_{i}\right) r_{i}\left\langle T_{r_{i}}\left(I-r_{i} A_{i}\right) x_{n}-x, A_{i} x-A_{i} x_{n}\right\rangle+2 \lambda^{2} \epsilon_{n} .
\end{aligned}
$$

Since $\left\{\alpha_{n}\right\}$ is strictly decreasing, $\delta_{n} \rightarrow 0, \epsilon_{n} \rightarrow 0, A_{i} x_{n}-A_{i} x \rightarrow 0$ and $\left\|x_{n}-x_{n+1}\right\| \rightarrow 0$, we have, for each $i \geq 1$,

$$
\left\|x_{n}-T_{r_{i}}\left(I-r_{i} A_{i}\right) x_{n}\right\| \longrightarrow 0, \quad n \longrightarrow \infty .
$$

Now, from $z_{n}-x_{n}=\sum_{i=1}^{n}\left(\alpha_{i-1}-\alpha_{i}\right)\left(T_{r_{i}} x_{n}-x_{n}\right)$ we get

$$
\left\|z_{n}-x_{n}\right\| \leq \sum_{i=1}^{n}\left(\alpha_{i-1}-\alpha_{i}\right)\left\|T_{r_{i}} x_{n}-x_{n}\right\|
$$

Since $\left\|x_{n}-T_{r_{i}} x_{n}\right\| \rightarrow 0$ and $0<\alpha_{i-1}-\alpha_{i}$ for each $i \geq 1$, one has

$$
\left\|z_{n}-x_{n}\right\| \longrightarrow 0, \quad \text { as } n \longrightarrow \infty
$$

Step 5. $\limsup _{n \rightarrow \infty}\left\langle(\gamma f-A) x^{*}, x_{n}-x^{*}\right\rangle \leq 0$.

To prove this, we pick a subsequence $\left\{x_{n_{j}}\right\}$ of $\left\{x_{n}\right\}$ such that

$$
\limsup _{n \rightarrow \infty}\left\langle(\gamma f-A) x^{*}, x_{n}-x^{*}\right\rangle=\lim _{j \rightarrow \infty}\left\langle(\gamma f-A) x^{*}, x_{n_{j}}-x^{*}\right\rangle .
$$

Without loss of generality, we may further assume that $x_{n_{j}} \rightarrow \widehat{x}$. Obviously, to prove Step 5 , we only need to prove that $\widehat{x} \in \Omega$.

Indeed, for each $i \geq 1$, since $x_{n}-T_{r_{i}}\left(I-r_{i} A_{i}\right) x_{n} \rightarrow 0, x_{n_{j}} \rightarrow \widehat{x}$ and $T_{r_{i}}\left(I-r_{i} A_{i}\right)$ is nonexpansive, by demiclosed principle of nonexpansive mapping we have

$$
\widehat{x} \in F\left(T_{r_{i}}\left(I-r_{i} A_{i}\right)\right)=E P\left(G_{i}, A_{i}\right), \quad i \geq 1 .
$$

Assume that $\lambda_{n_{m}, k} \rightarrow \lambda_{k} \in(0,1)$ for each $k=1,2, \ldots, N$. Let $W$ be the $W$-mapping generated by $T_{1}, \ldots, T_{N}$ and $\lambda_{1}, \ldots, \lambda_{N}$. Then, by Lemma 2.8 , we have

$$
W_{n_{m}} x \longrightarrow W x, \quad \forall x \in H
$$


Moreover, it follows from Lemma 2.7 that $\bigcap_{n=1}^{N} F\left(T_{i}\right)=F(W)$. Assume that $\hat{x} \notin F(W)$. Then $\widehat{x} \neq W \widehat{x}$. Since $\widehat{x} \in F\left(T_{r_{i}}\left(I-r_{i} A_{i}\right)\right)$ for each $i \geq 1$, by Step 3, (3.44) and Opial's property of the Hilbert space $H$, we have

$$
\begin{aligned}
& \liminf _{n \rightarrow \infty}\left\|x_{n_{m}}-\hat{x}\right\| \\
& \quad<\liminf _{n \rightarrow \infty}\left\|x_{n_{m}}-W \widehat{x}\right\| \\
& \leq \liminf _{n \rightarrow \infty}\left(\left\|x_{n_{m}}-W_{n_{m}} z_{n_{m}}\right\|+\left\|W_{n_{m}} z_{n_{m}}-W_{n_{m}} \widehat{x}\right\|+\left\|W_{n_{m}} \widehat{x}-W \widehat{x}\right\|\right) \\
& \leq \liminf _{n \rightarrow \infty}\left(\left\|x_{n_{m}}-W_{n_{m}} z_{n_{m}}\right\|+\left\|z_{n_{m}}-\widehat{x}\right\|+\left\|W_{n_{m}} \widehat{x}-W \widehat{x}\right\|\right) \\
& \leq \liminf _{n \rightarrow \infty}\left(\left\|x_{n_{m}}-W_{n_{m}} z_{n_{m}}\right\|+\left\|z_{n_{m}}-x_{n_{m}}\right\|+\left\|x_{n_{m}}-T_{r_{i}}\left(I-r_{i} A_{i}\right) x_{n_{m}}\right\|\right. \\
&\left.\quad+\left\|\widehat{x}-T_{r_{i}}\left(I-r_{i} A_{i}\right) x_{n_{m}}\right\|+\left\|W_{n_{m}} \widehat{x}-W \widehat{x}\right\|\right) \\
& \leq \liminf _{n \rightarrow \infty}\left(\left\|x_{n_{m}}-W_{n_{m}} z_{n_{m}}\right\|+\left\|z_{n_{m}}-x_{n_{m}}\right\|+\left\|x_{n_{m}}-T_{r_{i}}\left(I-r_{i} A_{i}\right) x_{n_{m}}\right\|\right. \\
&\left.\quad+\left\|T_{r_{i}}\left(I-r_{i} A_{i}\right) \widehat{x}-T_{r_{i}}\left(I-r_{i} A_{i}\right) x_{n_{m}}\right\|+\left\|W_{n_{m}} \widehat{x}-W \widehat{x}\right\|\right) \\
& \leq \liminf _{n \rightarrow \infty}\left\|x_{n_{m}}-\widehat{x}\right\|,
\end{aligned}
$$

which is a contradiction. Therefore, $\widehat{x} \in F(W)$. Hence, $\widehat{x} \in \Omega=\left(\bigcap_{i=1}^{N} F\left(T_{i}\right)\right) \cap\left(\bigcap_{i=1}^{\infty} E P\left(G_{i}, A_{i}\right)\right)$.

Step 6 . The sequence $\left\{x_{n}\right\}$ strongly converges to some point $x^{*} \in H$.

By using Lemmas 2.3 and 2.4, we have

$$
\begin{aligned}
\left\|x_{n+1}-x^{*}\right\|^{2}= & \left\|\left(I-\delta_{n} B-\epsilon_{n} A\right)\left(W_{n} z_{n}-x^{*}\right)+\delta_{n}\left(B x_{n}-B x^{*}\right)+\epsilon_{n}\left(\gamma f\left(x_{n}\right)-A x^{*}\right)\right\|^{2} \\
\leq & \left\|\left(I-\delta_{n} B-\epsilon_{n} A\right)\left(W_{n} z_{n}-x^{*}\right)+\delta_{n}\left(B x_{n}-B x^{*}\right)\right\|^{2} \\
& +2 \epsilon_{n}\left\langle\gamma f\left(x_{n}\right)-A x^{*}, x_{n+1}-x^{*}\right\rangle \\
\leq & {\left[\left(1-\delta_{n}\|B\|-\epsilon_{n} \bar{\gamma}\right)\left\|z_{n}-x^{*}\right\|+\delta_{n}\|B\|\left\|x_{n}-x^{*}\right\|\right]^{2} } \\
& +2 \epsilon_{n}\left\langle\gamma f\left(x_{n}\right)-A x^{*}, x_{n+1}-x^{*}\right\rangle \\
\leq & {\left[\left(1-\delta_{n}\|B\|-\epsilon_{n} \bar{\gamma}\right)\left\|x_{n}-x^{*}\right\|+\delta_{n}\|B\|\left\|x_{n}-x^{*}\right\|\right]^{2} } \\
& +2 \epsilon_{n}\left\langle\gamma f\left(x_{n}\right)-A x^{*}, x_{n+1}-x^{*}\right\rangle \\
= & {\left[\left(1-\epsilon_{n} \bar{\gamma}\right)\left\|x_{n}-x^{*}\right\|\right]^{2}+2 \epsilon_{n}\left\langle\gamma f\left(x_{n}\right)-A x^{*}, x_{n+1}-x^{*}\right\rangle } \\
\leq & \left(1-\epsilon_{n} \bar{\gamma}\right)^{2}\left\|x_{n}-x^{*}\right\|^{2}+2 \epsilon_{n} \gamma \kappa\left\|x_{n}-x^{*}\right\|\left\|x_{n+1}-x^{*}\right\| \\
& +2 \epsilon_{n}\left\langle\gamma f\left(x^{*}\right)-A x^{*}, x_{n+1}-x^{*}\right\rangle \\
\leq & \left(1-\epsilon_{n} \bar{\gamma}\right)^{2}\left\|x_{n}-x^{*}\right\|^{2}+\epsilon_{n} \gamma \kappa\left(\left\|x_{n}-x^{*}\right\|^{2}+\left\|x_{n+1}-x^{*}\right\|^{2}\right) \\
& +2 \epsilon_{n}\left\langle\gamma f\left(x^{*}\right)-A x^{*}, x_{n+1}-x^{*}\right\rangle,
\end{aligned}
$$


which implies that

$$
\begin{aligned}
\| x_{n+1}- & x^{*} \|^{2} \\
\leq & \frac{\left(1-\epsilon_{n} \bar{\gamma}\right)^{2}+\epsilon_{n} \gamma \kappa}{1-\epsilon_{n} \gamma \kappa}\left\|x_{n}-x^{*}\right\|^{2}+\frac{2 \epsilon_{n}}{1-\epsilon_{n} \gamma \kappa}\left\langle\gamma f\left(x^{*}\right)-A x^{*}, x_{n+1}-x^{*}\right\rangle \\
= & \frac{\left(1-2 \epsilon_{n} \bar{\gamma}+\epsilon_{n} \gamma \kappa\right)}{1-\epsilon_{n} \gamma \kappa}\left\|x_{n}-x^{*}\right\|^{2}+\frac{\epsilon_{n}^{2} \bar{\gamma}^{2}}{1-\epsilon_{n} \gamma \kappa}\left\|x_{n}-x^{*}\right\|^{2}+\frac{2 \epsilon_{n}}{1-\epsilon_{n} \gamma \kappa}\left\langle\gamma f\left(x^{*}\right)-A x^{*}, x_{n+1}-x^{*}\right\rangle \\
\leq & {\left[1-\frac{2 \epsilon_{n}(\bar{\gamma}-\kappa \gamma)}{1-\epsilon_{n} \gamma \kappa}\right]\left\|x_{n}-x^{*}\right\|^{2} } \\
& +\frac{2 \epsilon_{n}(\bar{\gamma}-\kappa \gamma)}{1-\epsilon_{n} \gamma \kappa}\left[\frac{1}{\bar{\gamma}-\kappa \gamma}\left\langle\gamma f\left(x^{*}\right)-A x^{*}, x_{n+1}-x^{*}\right\rangle+\frac{\epsilon_{n} \bar{\gamma}^{2}}{2(\bar{\gamma}-\kappa \gamma)} M^{\prime}\right],
\end{aligned}
$$

where $M^{\prime}$ is an appropriate constant such that $M^{\prime}=\sup _{n \geq 1}\left\{\left\|x_{n}-x^{*}\right\|\right\}$. Put

$$
\begin{gathered}
s_{n}=\frac{2 \epsilon_{n}(\bar{\gamma}-\kappa \gamma)}{1-\epsilon_{n} \kappa \gamma}, \\
t_{n}=\frac{1}{\bar{\gamma}-\kappa \gamma}\left\langle\gamma f\left(x^{*}\right)-A x^{*}, x_{n+1}-x^{*}\right\rangle+\frac{\epsilon_{n} \bar{\gamma}^{2}}{2(\bar{\gamma}-\kappa \gamma)} M^{\prime} .
\end{gathered}
$$

Then we have

$$
\left\|x_{n+1}-x^{*}\right\|^{2} \leq\left(1-s_{n}\right)\left\|x_{n}-x^{*}\right\|+s_{n} t_{n} .
$$

It follows from the assumption condition (E1) and (3.42) that

$$
\lim _{n \rightarrow \infty} s_{n}=0, \quad \sum_{n=1}^{\infty} s_{n}=\infty, \quad \limsup _{n \rightarrow \infty} t_{n} \leq 0
$$

Thus, applying Lemma 2.5 to (3.49), it follows that $x_{n} \rightarrow x^{*}$ as $n \rightarrow \infty$. This completes the proof.

By Theorem 3.1, we have the following direct corollaries.

Corollary 3.2. Let $H$ be a Hilbert space and $C$ be a nonempty closed convex subset of $H$. Let $f$ : $H \rightarrow H$ be a contraction with coefficient $0<\kappa<1, A: H \rightarrow H$ be strongly positive linear bounded self-adjoint operator with coefficient $\bar{\gamma}>0,\left\{T_{n}\right\}_{n=1}^{N}: H \rightarrow H(N \geq 1)$ be a finite family of nonexpansive mappings, $G: C \times C \rightarrow \mathbb{R}$ be a bifunction satisfying (A1)-(A4), and $A_{0}: C \rightarrow H$ be an $\alpha$-inverse strongly monotone mapping such that $\Omega=\bigcap_{i=1}^{N} F\left(T_{i}\right) \cap E P(G, A) \neq \emptyset$. Let $\left\{\varepsilon_{n}\right\}$ and $\left\{\delta_{n}\right\}$ be two sequences in $(0,1),\left\{\lambda_{n, i}\right\}_{i=1}^{N}$ be a sequence in $[a, b]$ with $0<a \leq b<1, r$ be a number in $(0,2 \alpha)$, and $\left\{\alpha_{n}\right\}$ be a sequence $[0,1)$. Take a fixed number $\gamma>0$ with $0<\bar{\gamma}-\gamma \kappa<1$. Assume that 
(E1) $\lim _{n \rightarrow \infty} \epsilon_{n}=0$ and $\sum_{n=1}^{\infty} \varepsilon_{n}=\infty$;

(E2) $\lim _{n \rightarrow \infty}\left|\lambda_{n+1, i}-\lambda_{n, i}\right|=0$ for each $i=1,2, \ldots, N$;

(E3) $0 \leq \delta_{n}+\epsilon_{n} \leq 1$ for all $n \geq 1$;

(E4) $\left\{\delta_{n}\right\} \subset\left[0, \min \left\{c, 1 / \bar{\beta},\left(2\|B\|+\|B\|^{2}-\bar{\beta}+\sqrt{\left.\left(\bar{\beta}-\|B\|^{2}-2\|B\|\right)^{2}+8 \bar{\beta}\|B\|\right)} / 4 \bar{\beta}\|B\|\right\}\right)\right.$ with $c<1$

(E5) $\sum_{n=1}^{\infty}\left|\epsilon_{n+1}-\epsilon_{n}\right|<\infty, \sum_{n=1}^{\infty}\left|\alpha_{n+1}-\alpha_{n}\right|<\infty, \sum_{n=1}^{\infty}\left|\delta_{n+1}-\delta_{n}\right|<\infty$.

Then the sequence $\left\{x_{n}\right\}$ defined by (1.25) converges strongly to $x^{*} \in \Omega$, which is the unique solution of the variational inequality:

$$
x^{*}=P_{\Omega}(I-(A-\gamma f)) x^{*}
$$

Remark 3.3. In the proof process of Theorem 3.1, we do not use Suzuki's Lemma (see [27]), which was used by many others for obtaining $\left\|x_{n+1}-x_{n}\right\| \rightarrow 0$ as $n \rightarrow \infty$ (see $\left.[4,5,28]\right)$. The proof method of $\widehat{x} \in \bigcap_{i=1}^{\infty} E P\left(G_{i}, A_{i}\right)$ is simple and different with ones of others.

\section{Applications for Multiobjective Optimization Problem}

In this section, we study a kind of multiobjective optimization problem by using the result of this paper. That is, we will give an iterative algorithm of solution for the following multiobjective optimization problem with the nonempty set of solutions:

$$
\begin{aligned}
& \min h_{1}(x), \quad x \in C, \\
& \min h_{2}(x),
\end{aligned}
$$

where $h_{1}(x)$ and $h_{2}(x)$ are both the convex and lower semicontinuous functions defined on a closed convex subset of $C$ of a Hilbert space $H$.

We denote by $A$ the set of solutions of the problem (4.1) and assume that $A \neq \emptyset$. Also, we denote the sets of solutions of the following two optimization problems by $A_{1}$ and $A_{2}$, respectively,

$$
\min h_{1}(x), \quad x \in C,
$$

and

$$
\min h_{2}(x), \quad x \in C
$$

Note that, if we find a solution $x \in A_{1} \cap A_{2}$, then one must have $x \in A$ obviously. 
Now, let $G_{1}$ and $G_{2}$ be two bifunctions from $C \times C$ to $\mathbb{R}$ defined by

$$
G_{1}(x, y)=h_{1}(y)-h_{1}(x), \quad G_{2}(x, y)=h_{2}(y)-h_{2}(x), \quad \forall(x, y) \in C \times C,
$$

respectively. It is easy to see that $E P\left(G_{1}\right)=A_{1}$ and $E P\left(G_{2}\right)=A_{2}$, where $E P\left(G_{i}\right)$ denotes the set of solutions of the equilibrium problem:

$$
G_{i}(x, y) \geq 0, \quad \forall y \in C, i=1,2
$$

respectively. In addition, it is easy to see that $G_{1}$ and $G_{2}$ satisfy the conditions (A1)-(A4). Let $\left\{\alpha_{n}\right\}$ be a sequence in $(0,1)$ and $r_{1}, r_{2} \in(0,1)$. Define a sequence $\left\{x_{n}\right\}$ by

$$
\begin{gathered}
x_{1} \in H, \\
z_{n}=\alpha_{n} T_{r_{1}} x_{n}+\left(1-\alpha_{n}\right) T_{r_{2}} x_{n}, \\
x_{n+1}=\epsilon_{n} \gamma f\left(x_{n}\right)+\left(1-\epsilon_{n}\right) z_{n}, \quad \forall n \geq 1 .
\end{gathered}
$$

By Theorem 3.1 with $A=I, N=1, T_{1}=I$ and $\delta_{n}=0$ for all $n \geq 1$, the sequence $\left\{x_{n}\right\}$ converges strongly to a solution $x^{*}=P_{A_{1} \cap A_{2}} \gamma f\left(x^{*}\right)$, which is a solution of the multiobjective optimization problem (4.1).

\section{References}

[1] K. Goebel and W. A. Kirk, Topics in Metric Fixed Point Theory, vol. 28 of Cambridge Studies in Advanced Mathematics, Cambridge University Press, Cambridge, UK, 1990.

[2] H.-K. Xu, "Viscosity approximation methods for nonexpansive mappings," Journal of Mathematical Analysis and Applications, vol. 298, no. 1, pp. 279-291, 2004.

[3] G. Marino and H.-K. Xu, "A general iterative method for nonexpansive mappings in Hilbert spaces," Journal of Mathematical Analysis and Applications, vol. 318, no. 1, pp. 43-52, 2006.

[4] Y. Yao, "A general iterative method for a finite family of nonexpansive mappings," Nonlinear Analysis: Theory, Methods E Applications, vol. 66, no. 12, pp. 2676-2687, 2007.

[5] V. Colao, G. Marino, and H.-K. Xu, "An iterative method for finding common solutions of equilibrium and fixed point problems," Journal of Mathematical Analysis and Applications, vol. 344, no. 1, pp. 340$352,2008$.

[6] S. Takahashi and W. Takahashi, "Viscosity approximation methods for equilibrium problems and fixed point problems in Hilbert spaces," Journal of Mathematical Analysis and Applications, vol. 331, no. 1, pp. 506-515, 2007.

[7] S. Plubtieng and R. Punpaeng, "A general iterative method for equilibrium problems and fixed point problems in Hilbert spaces," Journal of Mathematical Analysis and Applications, vol. 336, no. 1, pp. 455$469,2007$.

[8] L.-C. Ceng and J.-C. Yao, "Hybrid viscosity approximation schemes for equilibrium problems and fixed point problems of infinitely many nonexpansive mappings," Applied Mathematics and Computation, vol. 198, no. 2, pp. 729-741, 2008.

[9] L.-C. Ceng, S. Al-Homidan, Q. H. Ansari, and J.-C. Yao, "An iterative scheme for equilibrium problems and fixed point problems of strict pseudo-contraction mappings," Journal of Computational and Applied Mathematics, vol. 223, no. 2, pp. 967-974, 2009.

[10] L. C. Ceng, A. Petruşel, and J. C. Yao, "Iterative approaches to solving equilibrium problems and fixed point problems of infinitely many nonexpansive mappings," Journal of Optimization Theory and Applications, vol. 143, no. 1, pp. 37-58, 2009.

[11] S.-S. Chang, Y. J. Cho, and J. K. Kim, “Approximation methods of solutions for equilibrium problem in Hilbert spaces," Dynamic Systems and Applications, vol. 17, no. 3-4, pp. 503-513, 2008. 
[12] S. Chang, H. W. Joseph Lee, and C. K. Chan, "A new method for solving equilibrium problem fixed point problem and variational inequality problem with application to optimization," Nonlinear Analysis: Theory, Methods \& Applications, vol. 70, no. 9, pp. 3307-3319, 2009.

[13] P. Kumam and P. Katchang, "A viscosity of extragradient approximation method for finding equilibrium problems, variational inequalities and fixed point problems for nonexpansive mappings," Nonlinear Analysis: Hybrid Systems, vol. 3, no. 4, pp. 475-486, 2009.

[14] A. Moudafi, "Weak convergence theorems for nonexpansive mappings and equilibrium problems," Journal of Nonlinear and Convex Analysis, vol. 9, no. 1, pp. 37-43, 2008.

[15] S. Plubtieng and R. Punpaeng, "A new iterative method for equilibrium problems and fixed point problems of nonexpansive mappings and monotone mappings," Applied Mathematics and Computation, vol. 197, no. 2, pp. 548-558, 2008.

[16] X. Qin, Y. J. Cho, and S. M. Kang, "Viscosity approximation methods for generalized equilibrium problems and fixed point problems with applications," Nonlinear Analysis: Theory, Methods $\mathcal{E}$ Applications, vol. 72, no. 1, pp. 99-112, 2010.

[17] X. Qin, S. Y. Cho, and S. M. Kang, "Strong convergence of shrinking projection methods for quasi- $\phi$ nonexpansive mappings and equilibrium problems," Journal of Computational and Applied Mathematics, vol. 234, no. 3, pp. 750-760, 2010.

[18] S. H. Wang, G. Marino, and F. H. Wang, "Strong convergence theorems for a generalized equilibrium problem with a relaxed monotone mapping and a countable family of nonexpansive mappings in a hilbert space," Fixed Point Theory and Applications, vol. 2010, Article ID 230304, p. 22, 2010.

[19] S. H. Wang, Y. J. Cho, and X. L. Qin, "A new iterative method for solving equilibrium problems and fixed point problems for infinite family of nonexpansive mappings," Fixed Point Theory and Applications, vol. 2010, Article ID 165098, p. 18, 2010.

[20] H. Iiduka and W. Takahashi, "Strong convergence theorems for nonexpansive mappings and inversestrongly monotone mappings," Nonlinear Analysis: Theory, Methods E Applications, vol. 61, no. 3, pp. 341-350, 2005.

[21] W. Takahashi, Nonlinear Functional Analysis, Fixed Point Theory and Its Application, Yokohama Publishers, Yokohama, Japan, 2000.

[22] H.-K. Xu, "Iterative algorithms for nonlinear operators," Journal of the London Mathematical Society, vol. 66, no. 1, pp. 240-256, 2002.

[23] P. L. Combettes and S. A. Hirstoaga, "Equilibrium programming in Hilbert spaces," Journal of Nonlinear and Convex Analysis, vol. 6, no. 1, pp. 117-136, 2005.

[24] W. Takahashi, "Weak and strong convergence theorems for families of nonexpansive mappings and their applications," Annales Universitatis Mariae Curie-Skłodowska, vol. 51, no. 2, pp. 277-292, 1997.

[25] W. Takahashi and K. Shimoji, "Convergence theorems for nonexpansive mappings and feasibility problems," Mathematical and Computer Modelling, vol. 32, no. 11-13, pp. 1463-1471, 2000.

[26] S. Atsushiba and W. Takahashi, "Strong convergence theorems for a finite family of nonexpansive mappings and applications," Indian Journal of Mathematics, vol. 41, no. 3, pp. 435-453, 1999.

[27] T. Suzuki, "Strong convergence of Krasnoselskii and Mann's type sequences for one-parameter nonexpansive semigroups without Bochner integrals," Journal of Mathematical Analysis and Applications, vol. 305, no. 1, pp. 227-239, 2005.

[28] Y. J. Cho and X. Qin, "Convergence of a general iterative method for nonexpansive mappings in Hilbert spaces," Journal of Computational and Applied Mathematics, vol. 228, no. 1, pp. 458-465, 2009. 